PROCEEDINGS OF THE

AMERICAN MATHEMATICAL SOCIETY

Volume 134, Number 12, December 2006, Pages 3543-3553

S 0002-9939(06)08372-9

Article electronically published on May 31, 2006

\title{
THE KODAIRA DIMENSION OF DIFFEOMORPHIC KÄHLER THREEFOLDS
}

\author{
RAREŞ RĂSDEACONU
}

(Communicated by Jon G. Wolfson)

\begin{abstract}
We provide infinitely many examples of pairs of diffeomorphic, non-simply-connected Kähler manifolds of complex dimension three with different Kodaira dimensions. Also, in any possible Kodaira dimension we find infinitely many pairs of non-deformation equivalent, diffeomorphic Kähler threefolds.
\end{abstract}

\section{INTRODUCTION}

Let $M$ be a compact complex manifold of complex dimension $n$. On any such manifold the canonical line bundle $K_{M}=\wedge^{n, 0}$ encodes important information about the complex structure. One can define a series of birational invariants of $M, P_{k}(M):=h^{0}\left(M, K_{M}^{\otimes k}\right), k \geq 0$, called the plurigenera. The number of independent holomorphic $n$-forms on $M, p_{g}(M)=P_{1}(M)$ is called the geometric genus. The Kodaira dimension $\operatorname{Kod}(M)$ is a birational invariant given by

$$
\operatorname{Kod}(M)=\limsup \frac{\log h^{0}\left(M, K_{M}^{\otimes k}\right)}{\log k} .
$$

This can be shown to coincide with the maximal complex dimension of the image of $M$ under the pluri-canonical maps, so that $\operatorname{Kod}(M) \in\{-\infty, 0,1, \ldots, n\}$. A compact complex $n$-manifold is said to be of general type if $\operatorname{Kod}(M)=n$. For Riemann surfaces, the classification with respect to the Kodaira dimension, $\operatorname{Kod}(M)=-\infty, 0$ or 1 , is equivalent to the one given by the genus, $g(M)=0,1$, and $\geq 2$, respectively.

An important question in differential geometry is to understand how the complex structures on a given complex manifold are related to the diffeomorphism type of the underlying smooth manifold or further, to the topological type of the underlying topological manifold. Shedding some light on this question is S. Donaldson's result on the "failure of the $h$-cobordism conjecture in dimension four". In this regard, he found a pair of non-diffeomorphic, $h$-cobordant, simply connected 4-manifolds. One of them was $\mathbb{C P}_{2} \# 9 \mathbb{C P}_{2}$, the blow-up of $\mathbb{C P}_{2}$ at nine appropriate points, and the other one was a certain properly elliptic surface. For us, an important feature of these two complex surfaces is the fact that they have different Kodaira dimensions. Later, R. Friedman and Z. Qin [10] went further and proved that actually,

Received by the editors November 7, 2004 and, in revised form, June 16, 2005.

2000 Mathematics Subject Classification. Primary 32J17; Secondary 19J10.

Key words and phrases. Whitehead torsion, s-cobordism, Kodaira dimension, Kähler manifolds. 
for complex surfaces of Kähler type, the Kodaira dimension is invariant under diffeomorphisms. However, in higher dimensions, C. LeBrun and F. Catanese gave examples 4] of pairs of diffeomorphic projective manifolds of complex dimensions $2 n$ with $n \geq 2$, and Kodaira dimensions $-\infty$ and $2 n$.

In this article we address the question of the invariance of the Kodaira dimension under diffeomorphisms in complex dimension 3. We obtain the expected negative result.

Theorem A. For any allowed pair of distinct Kodaira dimensions $\left(d, d^{\prime}\right)$, with the exception of $(-\infty, 0)$ and $(0,3)$, there exist infinitely many pairs of diffeomorphic Kähler threefolds $\left(M, M^{\prime}\right)$, having the same Chern numbers, but with $\operatorname{Kod}(M)=d$ and $\operatorname{Kod}\left(M^{\prime}\right)=d^{\prime}$, respectively.

Corollary 0.1. For Kähler threefolds, the Kodaira dimension is not a smooth invariant.

Our examples also provide negative answers to questions regarding the deformation types of Kähler threefolds. Recall that two manifolds $X_{1}$ and $X_{2}$ are called directly deformation equivalent if there exists a complex manifold $\mathcal{X}$, and a proper holomorphic submersion $\varpi: \mathcal{X} \rightarrow \Delta$ with $\Delta=\{|z|<1\} \subset \mathbb{C}$, such that $X_{1}$ and $X_{2}$ occur as fibers of $\varpi$. The deformation equivalence relation is the equivalence relation generated by direct deformation equivalence.

One can easily see that two deformation equivalent manifolds are orientedly diffeomorphic. For complex surfaces of Kähler type there were strong indications that the converse should also be true. R. Friedman and J. Morgan proved 8] that not only is the Kodaira dimension a smooth invariant, but the plurigenera is also. However, Manetti [16] exhibited examples of diffeomorphic complex surfaces of general type which were not deformation equivalent. An easy consequence of our Theorem $\mathrm{A}$ and of the deformation invariance of plurigenera for 3 -folds 14 is that in complex dimension 3 the situation is similar.

Corollary 0.2. For Kähler threefolds the deformation type does not coincide with the diffeomorphism type.

Actually, with a bit more work we can get:

Theorem B. In any possible Kodaira dimension, there exist infinitely many examples of pairs of diffeomorphic, non-deformation equivalent Kähler threefolds with the same Chern numbers.

The examples we use are Cartesian products of simply connected, $h$-cobordant complex surfaces with Riemann surfaces. The real six-manifolds obtained will therefore be $h$-cobordant. To prove that these six-manifolds are in fact diffeomorphic, we use the $s$-cobordism theorem, by showing that the obstruction to the triviality of the corresponding $h$-cobordism, the Whitehead torsion, vanishes. Similar examples were previously used by Y. Ruan [20] to find pairs of diffeomorphic symplectic 6-manifolds which are not symplectic deformation equivalent. However, to show that his examples are diffeomorphic, Ruan uses the classification (up to diffeomorphisms) of simply connected, real 6-manifolds [19]. This restricts Ruan's construction to the case of Cartesian products by 2-spheres, a result which would also follow from Smale's $h$-cobordism theorem.

Our examples are pairs of complex structures of Kähler type with the same Chern numbers. This should be contrasted with C. LeBrun's examples [15] of 
complex structures, mostly non-Kähler, with different Chern numbers on a given differentiable real manifold.

In our opinion, the novelty of this article is the use of the apparently forgotten $s$-Cobordism Theorem. This theorem is especially useful when combined with a theorem on the vanishing of the Whitehead group. For this, there nowaday exists strong results, due to F.T. Farrell and L. Jones [6].

In the next section, we will review the main tools we use to find our examples: $h$-cobordisms, the Whitehead group and its vanishing. In section 3 we recall few well-known generalities about complex surfaces. Sections 4 and 5 contain a number of examples and the proofs of Theorems $\mathrm{A}$ and $\mathrm{B}$. In the last section we conclude with a few remarks, and we raise some natural questions.

\section{THE $s$-COBORDISM THEOREM}

Definition 1.1. Let $M$ and $M^{\prime}$ be two $n$-dimensional smooth, compact, oriented manifolds. A cobordism between $M$ and $M^{\prime}$ is a triplet $\left(W ; M, M^{\prime}\right)$, where $W$ is an $(n+1)$-dimensional compact, oriented manifold with boundary, $\partial W=\overline{\partial W_{-}} \amalg \partial W_{+}$ with $\partial W_{-}=M$ and $\partial W_{+}=M^{\prime}$ (by $\overline{\partial W_{-}}$we denote the orientation-reversed version of $\left.\partial W_{-}\right)$.

We say that the cobordism $\left(W ; M, M^{\prime}\right)$ is an $h$-cobordism if the inclusions $i_{-}$: $M \rightarrow W$ and $i_{+}: M^{\prime} \rightarrow W$ are homotopy equivalences between $M, M^{\prime}$ and $W$.

The following well-known results [23, 24] allow us to easily check when two simply connected 4 -manifolds are $h$-cobordant.

Theorem 1.2. Two simply connected, compact, oriented smooth manifolds of dimension 4 are $h$-cobordant if and only if their intersection forms are isomorphic.

Theorem 1.3. Any indefinite, unimodular, bilinear form is uniquely determined by its rank, signature and parity.

In higher dimensions any $h$-cobordism $\left(W ; M, M^{\prime}\right)$ is controlled by the Whitehead torsion $\tau(W ; M)$, an element of the so-called Whitehead group, which will be defined below.

Let $\Pi$ be any group, and $R=\mathbb{Z}(\Pi)$ the ring generated by $\Pi$. We denote by $G L_{n}(R)$ the group of all non-singular $n \times n$ matrices over $R$. For all $n$ we have a natural inclusion $G L_{n}(R) \subset G L_{n+1}(R)$ identifying each $A \in G L_{n}(R)$ with the matrix

$$
\left(\begin{array}{cc}
A & 0 \\
0 & 1
\end{array}\right) \in G L_{n+1}(R) \text {. }
$$

Let $G L(R)=\cup_{n=1}^{\infty} G L_{n}(R)$. We define the following group:

$$
K_{1}(R)=G L(R) /[G L(R), G L(R)] .
$$

The Whitehead group we are interested in is:

$$
W h(\Pi)=K_{1}(R) /\langle \pm g \mid g \in \Pi\rangle .
$$

Theorem 1.4. Let $M$ be a smooth, compact, oriented manifold. For any $h$ cobordism $W$ of $M$ with $\partial_{-} W=M$, and with dim $W \geq 6$, there exists an element $\tau(W) \in W h\left(\pi_{1}(M)\right)$, called the Whitehead torsion, characterized by the following properties:

$s$-cobordism theorem: $\tau(W) \in W h\left(\pi_{1}(M)\right)=0$ if and only if the $h$ cobordism is trivial, i.e. $W$ is diffeomorphic to $\partial_{-} W \times[0,1]$. 
Existence: Given $\alpha \in W h\left(\pi_{1}(M)\right)$, there exists an $h$-cobordism $W$ with $\tau(W)=\alpha$.

Uniqueness: $\tau(W)=\tau\left(W^{\prime}\right)$ if and only if there exists a diffeomorphism $h: W \rightarrow W^{\prime}$ such that $h_{\mid M}=i d_{M}$.

For the definition of the Whitehead torsion and the above theorem we refer the interested reader to Milnor's article [18. When $M$ is simply connected, the $s$-cobordism theorem is nothing but the usual $h$-cobordism theorem [17, due to Smale.

This theorem will be a stepping stone in finding pairs of diffeomorphic manifolds in dimensions greater than 5, provided there is knowledge about the vanishing of the Whitehead groups. The most powerful vanishing theorem that we are aware of is the following.

Theorem 1.5 (Farrell, Jones). Let $M$ be a compact Riemannian manifold of nonpositive sectional curvature. Then $W h\left(\pi_{1}(M)\right)=0$.

The uniformization theorem of compact Riemann surfaces then yields the following result which, as was kindly pointed out to us by L. Jones, was also known to F. Waldhausen [22] long before 6].

Corollary 1.6. Let $\Sigma$ be a compact Riemann surface. Then $W h\left(\pi_{1}(\Sigma)\right)=0$.

A useful corollary, which will be frequently used, is the following.

Corollary 1.7. Let $M$ and $M^{\prime}$ be two simply connected, h-cobordant 4-manifolds, and let $\Sigma$ be a Riemann surface. Then $M \times \Sigma$ and $M^{\prime} \times \Sigma$ are diffeomorphic.

Proof. Let $W$ be an $h$-cobordism between $M$ and $M^{\prime}$ such that $\partial_{-} W=M$ and $\partial_{+} W=M^{\prime}$ and let $\widetilde{W}=W \times \Sigma$. Then $\partial_{-} \widetilde{W}=M \times \Sigma, \partial_{+} \widetilde{W}=M^{\prime} \times \Sigma$, and $\widetilde{W}$ is an $h$-cobordism between $M \times \Sigma$ and $M^{\prime} \times \Sigma$. Now, since $M$ is simply connected, $\pi_{1}(M \times \Sigma)=\pi_{1}(\Sigma)$ and so $W h\left(\pi_{1}(M \times \Sigma)\right)=W h\left(\pi_{1}(\Sigma)\right)$. By the uniformization theorem any Riemann surface of positive genus admits a metric of non-positive curvature. Thus, by Theorem 1.5. $W h\left(\pi_{1}(\Sigma)\right)=0$, which, by the first property of Theorem 1.4, implies that $M \times \Sigma$ and $M^{\prime} \times \Sigma$ are diffeomorphic. When the genus of the Riemann surface is zero, the result similarly follows from Smale's $h$-cobordism theorem.

\section{Generalities}

To prove Theorems $\mathrm{A}$ and $\mathrm{B}$, we will use our Corollary 1.7, and take for $M$ and $M^{\prime}$ appropriate $h$-cobordant, simply connected, complex projective surfaces, endowed with their natural orientation, and for $\Sigma$, appropriate Riemann surfaces. To find examples of $h$-cobordant complex surfaces, we use:

Proposition 2.1. Let $M$ and $M^{\prime}$ be two simply connected complex surfaces with the same geometric genus $p_{g}, c_{1}^{2}(M)-c_{1}^{2}\left(M^{\prime}\right)=m \geq 0$ and let $k>0$ be any integer. Let $X$ be the blowing-up of $M$ at $k+m$ distinct points and let $X^{\prime}$ be the blowing-up of $M^{\prime}$ at $k$ distinct points. Then $X$ and $X^{\prime}$ are $h$-cobordant, $\operatorname{Kod}(X)=\operatorname{Kod}(M)$ and $\operatorname{Kod}\left(X^{\prime}\right)=\operatorname{Kod}\left(M^{\prime}\right)$.

Proof. By Noether's formula we see that $b_{2}\left(M^{\prime}\right)=b_{2}(M)+m$. Since, by blowing-up we increase the second Betti number by one each time, it follows that $b_{2}\left(X^{\prime}\right)=$ $b_{2}(X)$. Using the birational invariance of the plurigenera, we have that $b_{+}\left(X^{\prime}\right)=$ 
$2 p_{g}+1=b_{+}(X)$. As $X$ and $X^{\prime}$ are both non-spin, and their intersection forms have the same rank and signature, their intersection forms are isomorphic. Thus, by Theorem 1.2 $X$ and $X^{\prime}$ are $h$-cobordant. The statement about the Kodaira dimension follows from its birational invariance, too.

Corollary 2.2. Let $S$ and $S^{\prime}$ be two simply connected, $h$-cobordant complex surfaces. If $S_{k}$ and $S_{k}^{\prime}$ are the blowing-ups of the two surfaces, each at $k \geq 0$ distinct points, then $S_{k}$ and $S_{k}^{\prime}$ are $h$-cobordant, too. Moreover, $\operatorname{Kod}\left(S_{k}\right)=\operatorname{Kod}(S)$, and $\operatorname{Kod}\left(S_{k}^{\prime}\right)=\operatorname{Kod}\left(S^{\prime}\right)$.

The following proposition will take care of the computation of the Kodaira dimension of our examples. Its proof is standard, and we will omit it.

Proposition 2.3. Let $V$ and $W$ be two complex manifolds. Then $P_{m}(V \times W)=$ $P_{m}(V) \cdot P_{m}(W)$. In particular, $\operatorname{Kod}(V \times W)=\operatorname{Kod}(V)+\operatorname{Kod}(W)$.

For the computation of the Chern numbers of the examples involved, we need:

Proposition 2.4. Let $M$ be a smooth complex surface with $c_{1}^{2}(M)=a, c_{2}(M)=b$, and let $\Sigma$ be a smooth complex curve of genus $g$, and $X=M \times \Sigma$ their Cartesian product. The Chern numbers $\left(\mathbf{c}_{\mathbf{1}}^{\mathbf{3}}, \mathbf{c}_{\mathbf{1}} \mathbf{c}_{\mathbf{2}}, \mathbf{c}_{\mathbf{3}}\right)$ of $X$ are $((6-6 g) a,(2-2 g)(a+b)$, $(2-2 g) b)$.

Proof. Let $p: X \rightarrow M$, and $q: X \rightarrow \Sigma$ be the projections onto the two factors. Then the total Chern class is $c(X)=p^{*} c(M) \cdot q^{*} c(\Sigma)$, which allows us to identify the Chern classes. Integrating over $X$, the result immediately follows.

\section{Diffeomorphism types - Proof of Theorem A}

In this section we prove Theorem $\mathrm{A}$ To do this, for each of the pairs of Kodaira dimensions stated, we provide infinitely many examples, by taking Cartesian products of appropriate $h$-cobordant Kähler surfaces with Riemann surfaces of positive genus.

Example 1. Pairs of Kodaira dimensions $(-\infty, 1)$ and $(-\infty, 2)$.

Let $M$ be the blowing-up of $\mathbb{C P}_{2}$ at 9 distinct points given by the intersection of two generic cubics. $M$ is a non-spin, simply connected complex surface with $\operatorname{Kod}(M)=-\infty$ which is also an elliptic fibration, $\pi: M \rightarrow \mathbb{C P}_{1}$. By taking the cubics general enough, we may assume that $M$ has no multiple fibers, and the only singular fibers are irreducible curves with one ordinary double point. Let $M^{\prime}$ be obtained from $M$ by performing logarithmic transformations on two of its smooth fibers, with multiplicities $p$ and $q$, where $p$ and $q$ are two relatively prime positive integers. $M^{\prime}$ is also an elliptic surface, $\pi^{\prime}: M^{\prime} \rightarrow \mathbb{C P}_{1}$, whose fibers can be identified to those in $M$ except for the pair of multiple fibers $F_{1}$, and $F_{2}$. Let $F$ be the homology class of the generic fiber in $M^{\prime}$. In homology we have $[F]=p\left[F_{1}\right]=q\left[F_{2}\right]$. By the canonical bundle formula, we see that $K_{M}=-F$ and

$$
K_{M^{\prime}}=-F+(p-1) F_{1}+(q-1) F_{2}=\frac{p q-p-q}{p q} F .
$$

Then $p_{g}(M)=p_{g}\left(M^{\prime}\right)=0, c_{1}^{2}(M)=c_{1}^{2}\left(M^{\prime}\right)=0$, and $\operatorname{Kod}\left(M^{\prime}\right)=1$. Moreover, from [9, Theorem 2.3, page 158] $M^{\prime}$ is simply connected and non-spin.

For any $k \geq 0$, let $M_{k}$ and $M_{k}^{\prime}$ be the blowing-ups at $k$ distinct points of $M$ and $M^{\prime}$, respectively, and let $\Sigma$ be a Riemann surface. If $g(\Sigma)=1$, according to 
Corollary 1.7 and Proposition 2.3. $\left(M_{k} \times \Sigma_{1}, M_{k}^{\prime} \times \Sigma_{1}\right), k \geq 0$ will provide infinitely many pairs of diffeomorphic Kähler threefolds, of Kodaira dimensions $-\infty$ and 1 , respectively. If $g(\Sigma) \geq 2$, we get infinitely many pairs of diffeomorphic Kähler threefolds with Kodaira dimensions $-\infty$, and 2, respectively. The statement about the Chern numbers follows from Proposition 2.4.

Example 2. Pairs of Kodaira dimensions $(0,1)$ and $(0,2)$.

In $\mathbb{C P}_{1} \times \mathbb{C P}_{2}$, let $M$ be the the generic section of line bundle $p_{1}^{*} \mathcal{O}_{\mathbb{C P}_{1}}(2) \otimes$ $p_{2}^{*} \mathcal{O}_{\mathbb{C P}_{2}}(3)$, where $p_{i}, i=1,2$, are the projections onto the two factors. Then $M$ is a $K 3$ surface, i.e. a smooth, simply connected complex surface, with trivial canonical bundle. Moreover, using the projection onto the first factor, it fibers over $\mathbb{C P}_{1}$ with elliptic fibers.

Kodaira 12 produced infinitely many examples of properly elliptic surfaces of Kähler type, homotopically equivalent to a $K 3$ surface, by performing two logarithmic transformations on two smooth fibers with relatively prime multiplicities on such an elliptic $K 3$. Let $M^{\prime}$ to be any such surface, and let $M_{k}$ and $M_{k}^{\prime}$ be the blowing-ups at $k$ distinct points of $M$ and $M^{\prime}$, respectively. As before, let $\Sigma$ be a Riemann surface. If $g(\Sigma)=1$, the Cartesian products $M_{k} \times \Sigma$ and $M_{k}^{\prime} \times \Sigma$ will provide infinitely many pairs of diffeomorphic Kähler 3-folds of Kodaira dimensions 0 and 1 , respectively. If $g(\Sigma) \geq 2$, we obtain pairs in Kodaira dimensions 1 and 2, respectively. Again, the statement about the Chern numbers follows from Proposition 2.4.

Example 3. Pairs of Kodaira dimensions $(-\infty, 2)$ and $(-\infty, 3)$.

Arguing as before, we present a pair of simply connected, $h$-cobordant projective surfaces, one on Kodaira dimension 2, and the other one of Kodaira dimension $-\infty$.

Let $M$ be the Barlow surface [1]. This is a non-spin, simply connected projective surface of general type, with $p_{g}=0$ and $c_{1}^{2}(M)=1$. It is therefore $h$-cobordant to $M^{\prime}$, the projective plane $\mathbb{C P}_{2}$ blown-up at 8 points. By taking the Cartesian product of their blowing-ups by a Riemann surface of genus 1, we obtain diffeomorphic, projective threefolds of Kodaira dimensions 3 and $-\infty$, respectively. For a Riemann surface of bigger genus, we obtain diffeomorphic, projective threefolds of Kodaira dimensions 2 and $-\infty$, respectively. The invariance of their Chern numbers follows as usual.

Example 4. Pairs of Kodaira dimensions $(0,2)$ and $(1,3)$.

Following [3], we will describe an example of a simply connected, minimal surface of general type with $c_{1}^{2}=p_{g}=1$.

In $\mathbb{C P}_{2}$ we consider two generic smooth cubics $F_{1}$ and $F_{2}$, which meet transversally at 9 distinct points, $x_{1}, \ldots, x_{9}$, and let $\sigma: \tilde{X} \rightarrow \mathbb{C P}_{2}$ be the blowing-up of $\mathbb{C P}_{2}$ at $x_{1}, \ldots, x_{9}$, with exceptional divisors $\tilde{E}_{i}, i=1, \ldots, 9$. Let $\tilde{F}_{1}$ and $\tilde{F}_{2}$ be the strict transforms of $F_{1}$ and $F_{2}$, respectively. Then $\tilde{F}_{1}$ and $\tilde{F}_{2}$ are two disjoint, smooth divisors, and we can easily see that $\mathcal{O}_{\tilde{X}}\left(\tilde{F}_{1}+\tilde{F}_{2}\right)=\tilde{\mathcal{L}}^{\otimes 2}$, where

$$
\tilde{\mathcal{L}}=\sigma^{*} \mathcal{O}_{\mathbb{C P}_{2}}(3) \otimes \mathcal{O}_{\tilde{X}}\left(\tilde{E}_{1}+\cdots+\tilde{E}_{9}\right) .
$$

Let $\pi: \bar{X} \rightarrow \tilde{X}$ to be the double covering of $\tilde{X}$ branched along the smooth divisor $\tilde{F}_{1}+\tilde{F}_{2}$. We denote by $p: \bar{X} \rightarrow \mathbb{C P}_{2}$ the composition $\sigma \circ \pi$, and by $\bar{F}_{1}, \bar{F}_{2}$ the reduced divisors $\pi^{-1}\left(\tilde{F}_{1}\right)$, and $\pi^{-1}\left(\tilde{F}_{2}\right)$, respectively. Since each $\tilde{E}_{i}$ intersects the branch locus at 2 distinct points, we can see that for each $i=1, \ldots, 9, \bar{E}_{i}=\pi^{-1}\left(\tilde{E}_{i}\right)$ is a smooth (-2)-curve such that $\pi_{\mid \bar{E}_{i}}: \bar{E}_{i} \rightarrow \tilde{E}_{i}$ is the double covering of $\tilde{E}_{i}$ branched 
at the two intersection points of $\tilde{E}_{1}$ with $\tilde{F}_{1}+\tilde{F}_{2}$. As the $\tilde{E}_{i}$ 's are mutually disjoint, the $\bar{E}_{i}$ 's will also be mutually disjoint. Similarly, if $\ell$ is a line in $\mathbb{C P}_{2}$ not passing through any of the intersection points of $F_{1}$ with $F_{2}$, then $L=p^{*}(\ell)=p^{*} \mathcal{O}_{\mathbb{C P}_{2}}(1)$ is a smooth curve of genus 2 , not intersecting any of the $\bar{E}_{i}$ 's. Since

$$
p^{*} \mathcal{O}_{\mathbb{C P}_{2}}(3)=\mathcal{O}_{\bar{X}}\left(2 \bar{F}_{1}+\bar{E}_{1}+\cdots+\bar{E}_{9}\right),
$$

we can write as before $\mathcal{O}_{\bar{X}}\left(L+\bar{E}_{1}+\cdots+\bar{E}_{9}\right)=\overline{\mathcal{L}}^{\otimes 2}$, where

$$
\overline{\mathcal{L}}=p^{*} \mathcal{O}_{\mathbb{C P}_{2}}(2) \otimes \mathcal{O}_{\bar{X}}\left(-\bar{F}_{1}\right)
$$

Now let $\phi: \bar{S} \rightarrow \bar{X}$ be the double covering of $\bar{X}$ ramified along the smooth divisor $L+\bar{E}_{1}+\cdots+\bar{E}_{9}$. The surface $\bar{S}$ is non-minimal with exactly 9 disjoint exceptional curves of the first kind. Namely, the reduced divisors $\phi^{-1}\left(\bar{E}_{i}\right), i=$ $1, \ldots, 9$. The surface $S$ we were looking for is obtained from $\bar{S}$ by blowing down these 9 exceptional curves.

F. Catanese proves 3 , that $S$ is a simply connected, minimal surface of general type with $c_{1}^{2}(S)=p_{g}(S)=1$.

Let $S_{k}^{\prime}$ be the blowing-up of a $K 3$ surface at $k$ distinct points. Also let $S_{k}$ denote the blowing-up of $S$ at $k+1$ distinct points, and let $\Sigma$ be a Riemann surface. If $g(\Sigma)=1,\left(S_{k} \times \Sigma, S_{k}^{\prime} \times \Sigma\right)$ will provide infinitely many pairs of diffeomorphic Kähler threefolds of Kodaira dimensions 2 and 0 , respectively. If $g(\Sigma) \geq 2$ we get infinitely many pairs of diffeomorphic Kähler threefolds of Kodaira dimensions 3 and 1, respectively. The statement about the Chern classes follows as before.

Example 5. Pairs of Kodaira dimensions $(1,2)$ and $(2,3)$.

In $\mathbb{C P}_{1} \times \mathbb{C P}_{2}$, let $M_{n}$ be the the generic section of the line bundle $p_{1}^{*} \mathcal{O}_{\mathbb{C P}_{1}}(n) \otimes$ $p_{2}^{*} \mathcal{O}_{\mathbb{C P}_{2}}(3)$ for $n \geq 3$, where $p_{i}, i=1,2$, are the projections onto the two factors. Then $M_{n}$ is a smooth, simply connected projective surface, and using the projection onto the first factor we see that $M_{n}$ is a properly elliptic surface. By the adjunction formula, the canonical line bundle is

$$
K_{M_{n}}=p_{1}^{*} \mathcal{O}_{\mathbb{C P}_{1}}(n-2) .
$$

From this and the projection formula we can find the purigenera:

$$
\begin{aligned}
P_{m}\left(M_{n}\right) & =h^{0}\left(M_{n}, K_{M_{n}}^{\otimes m}\right)=h^{0}\left(M_{n}, p_{1}^{*} \mathcal{O}_{\mathbb{C P}_{1}}(m(n-2))\right) \\
& =h^{0}\left(\mathbb{C P}_{1}, p_{1 *} p_{1}^{*} \mathcal{O}_{\mathbb{C P}_{1}}(m(n-2))\right) \\
& =h^{0}\left(\mathbb{C P}_{1}, \mathcal{O}_{\mathbb{C P}_{1}}(m(n-2))\right) \\
& =m(n-2)+1 .
\end{aligned}
$$

So, $\operatorname{Kod}\left(M_{n}\right)=1$, and $p_{g}\left(M_{n}\right)=n-1$. We can also see that $c_{1}^{2}\left(M_{n}\right)=0$.

Let $M^{\prime}$ be any smooth sextic in $\mathbb{C P}_{3} . M^{\prime}$ is a simply connected surface of general type with $p_{g}\left(M^{\prime}\right)=10$, and $c_{1}^{2}\left(M^{\prime}\right)=24$. Let $M_{k}^{\prime}$ be the blowing-up of $M^{\prime}$ at $24+k$ distinct points, let $M_{k}$ be the blowing-up of $M_{11}$ at $k+1$ points, and let $\Sigma$ be a Riemann surface. If $g(\Sigma)=1,\left(M_{k} \times \Sigma, M_{k}^{\prime} \times \Sigma\right)$ will provide infinitely many pairs of diffeomorphic Kähler threefolds of Kodaira dimensions 1 and 2, respectively, while if $g(\Sigma) \geq 2$ we get infinitely many pairs of diffeomorphic Kähler threefolds of Kodaira dimensions 2 and 3, respectively. The statement about the Chern classes again follows. 


\section{Deformation type - Proof of Theorem B}

Similar idea is used to prove Theorem $B$. The proof follows from the examples below.

Example 1. Kodaira dimension $-\infty$.

Here we again use the Barlow surface $M$, and $M^{\prime}$ the blowing-up of $\mathbb{C P}_{2}$ at 8 points as two $h$-cobordant complex surfaces. Let $S_{k}$ and $S_{k}^{\prime}$ denote the blowing-ups of $M$ and $M^{\prime}$, respectively at $k$ distinct points. Then, by the classical $h$-cobordism theorem, $X_{k}=S_{k} \times \mathbb{C P}_{1}$ and $X_{k}^{\prime}=S_{k}^{\prime} \times \mathbb{C P}_{1}$ are two diffeomorphic 3 -folds with the same Kodaira dimension $-\infty$. The fact that $X_{k}$ and $X_{k}^{\prime}$ are not deformation equivalent follows as in [20] from Kodaira's stability theorem [13]. We also see immediately that they have the same Chern numbers.

Example 2. Kodaira dimension 2 and 3.

We start with a Horikawa surface, namely a simply connected surface of general type $M$ with $c_{1}^{2}(M)=16$ and $p_{g}(M)=10$. An example of such a surface can be obtained as a ramified double cover of $Y=\mathbb{C P}_{1} \times \mathbb{C P}_{1}$ branched at a generic curve of bi-degree $(6,12)$. Let $p: M \rightarrow Y$ denote its degree 2 morphism onto $Y$. The canonical bundle of $M$ is $K_{M}=\mathcal{O}_{Y}(1,4)$; see [2, page 182]. Here by $\mathcal{O}_{Y}(a, b)$ we denote the line bundle $p_{1}^{*} \mathcal{O}_{\mathbb{C P}_{1}}(a) \otimes p_{2}^{*} \mathcal{O}_{\mathbb{C P}_{1}}(b)$, where $p_{i}, 1=1,2$, are the projections of $Y$ onto the two factors. Note that the formula for the canonical bundle shows that $M$ is not spin.

Lemma 4.1. The plurigenera of $M$ are given by

$$
P_{n}(M)=\left\{\begin{array}{cc}
10, & n=1, \\
8 n^{2}-8 n+11, & n \geq 2,
\end{array}\right.
$$

Proof. (cf. 2] ) We have $p_{*} \mathcal{O}_{M}=\mathcal{O}_{Y} \oplus \mathcal{O}_{Y}(-3,-6)$. We have:

$$
\begin{aligned}
P_{n}(M) & =h^{0}\left(M, p^{*} \mathcal{O}_{Y}(n, 4 n)\right)=h^{0}\left(Y, p_{*} p^{*} \mathcal{O}_{Y}(n, 4 n)\right) \\
& =h^{0}\left(Y, \mathcal{O}_{Y}(n, 4 n) \otimes p_{*} \mathcal{O}_{M}\right) \\
& =h^{0}\left(Y, \mathcal{O}_{Y}(n, 4 n)\right)+h^{0}\left(Y, \mathcal{O}_{Y}(n-3,4 n-6)\right) .
\end{aligned}
$$

Now, if $n<3$ we get $P_{n}(M)=(n+1)(4 n+1)$. In particular, $p_{g}(M)=10$ and $P_{2}(M)=27$. If $n \geq 3, P_{n}(M)=(n+1)(4 n+1)+(n-2)(4 n-5)=8 n^{2}-8 n+11$.

Let $M^{\prime} \subset \mathbb{C P}_{3}$ be a smooth sextic. The adjunction formula will again provide the the canonical bundle $K_{M^{\prime}}=\mathcal{O}_{M^{\prime}}(2)$, and so $c_{1}^{2}\left(M^{\prime}\right)=24$.

Lemma 4.2. The plurigenera of $M^{\prime}$ are given by

$$
P_{n}\left(M^{\prime}\right)=\left\{\begin{array}{cc}
\left(\begin{array}{c}
2 n+3 \\
3
\end{array}\right), & n=1,2, \\
12 n^{2}-12 n+11, & n \geq 3 .
\end{array}\right.
$$

Proof. From the exact sequence $0 \rightarrow \mathcal{O}_{\mathbb{C P}_{3}}(2 n-6) \rightarrow \mathcal{O}_{\mathbb{C P}_{3}}(2 n) \rightarrow K_{M^{\prime}}^{\otimes n} \rightarrow 0$, we get

$$
\begin{aligned}
0 & \rightarrow H^{0}\left(\mathbb{C P}_{3}, \mathcal{O}_{\mathbb{C P}_{3}}(2 n-6)\right) \rightarrow H^{0}\left(\mathbb{C P}_{3}, \mathcal{O}_{\mathbb{C P}_{3}}(2 n)\right) \\
& \rightarrow H^{0}\left(M^{\prime}, K_{M^{\prime}}^{\otimes n}\right) \rightarrow H^{1}\left(\mathbb{C P}_{3}, \mathcal{O}_{\mathbb{C P}_{3}}(2 n)\right)=0
\end{aligned}
$$

So, for $n \geq 3$,

$$
P_{n}\left(M^{\prime}\right)=\left(\begin{array}{c}
2 n+3 \\
3
\end{array}\right)-\left(\begin{array}{c}
2 n-3 \\
3
\end{array}\right)=12 n^{2}-12 n+11
$$


while for $n<3, P_{n}\left(M^{\prime}\right)=\left(\begin{array}{c}2 n+3 \\ 3\end{array}\right)$. In particular, $p_{g}\left(M^{\prime}\right)=10$ and $P_{2}\left(M^{\prime}\right)=$ 35 .

Let $M_{k}$ be the blowing-up of $M$ at $k$ distinct points, let $M_{k}^{\prime}$ be the blowing-up of $M^{\prime}$ at $8+k$ distinct points, and let $\Sigma$ be a Riemann surface. If $g(\Sigma)=1$, then $\left(M_{k} \times \Sigma, M_{k}^{\prime} \times \Sigma\right), k \geq 0$, will provide the required examples of Kodaira dimension 2 , and if $g(\Sigma) \geq 2$, it will provide the required examples of Kodaira dimension 3 .

To prove that they are not deformation equivalent we will use the deformation invariance of the plurigenera theorem [14, page 535]. Because of the their multiplicative property (cf. Proposition 2.3), it will suffice to look at the plurigenera of $M$ and $M^{\prime}$. But, $P_{2}(M)=27$ and $P_{2}\left(M^{\prime}\right)=P_{2}(S)=35$, and so $M \times \Sigma$ and $M^{\prime} \times \Sigma$ are not deformation equivalent.

The statement about the Chern numbers of this example follows immediately.

Example 3. Kodaira dimension 1.

Here we use again the elliptic surfaces $\pi: M_{p, q} \rightarrow \mathbb{C P}_{1}$ obtained from the rational elliptic surface by applying logarithmic transformations on two smooth fibers, with relatively prime multiplicities $p$ and $q$. From (3.1) we get $K_{M_{p, q}}^{\otimes p q}=$ $p^{*} \mathcal{O}_{\mathbb{C P}_{1}}((p q-p-q))$. Hence $P_{p q}\left(M_{p, q}\right)=p q-p-q+1$, while if $n \leq p q, P_{n}\left(M_{p, q}\right)=0$, the class of $F$ being a primitive element in $H^{2}\left(M_{p, q}, \mathbb{Z}\right)$; cf. [12. It is easy to see now that, for example, if $(p, q) \neq(2,3), P_{6}\left(M_{p, q}\right) \neq P_{6}\left(M_{2,3}\right)$. If $\Sigma$ is any smooth elliptic curve, the 3 -folds $X_{p, q}=M_{p, q} \times \Sigma$ will provide infinitely many diffeomorphic Kähler threefolds of Kodaira dimension 1. Proposition 2.4 again shows that all these threefolds have the same Chern numbers. The above computation of plurigenera shows that, in general, the $X_{p, q}$ 's have different plurigenera. Hence, these Kähler threefolds are not deformation equivalent.

\section{Example 4. Kodaira dimension 0.}

Here we are supposed to start with a simply connected minimal surface of zero Kodaira dimension. But, up to diffeomorphisms there exists only one [2], the $K 3$ surface. So our method fails to produce examples in this case. However, M. Gross constructed 11] a pair of diffeomorphic complex threefolds with trivial canonical bundle, which are not deformation equivalent. For the sake of completeness we will briefly recall his examples.

Let $E_{1}=\mathcal{O}_{\mathbb{C P}_{1}}^{\oplus 4}$ and $E_{2}=\mathcal{O}_{\mathbb{C P}_{1}}(-1) \oplus \mathcal{O}_{\mathbb{C P}_{1}}(1) \oplus \mathcal{O}_{\mathbb{C P}_{1}}^{\oplus 2}$ be the two rank 4 vector bundles over $\mathbb{C P}_{1}$, and consider $X_{1}=\mathbb{P}\left(E_{1}\right)$ and $X_{2}=\mathbb{P}\left(E_{2}\right)$ their projectivizations. Note that $E_{2}$ deforms to $E_{1}$. Let $M_{i} \in\left|-K_{X_{i}}\right|, i=1,2$, be general anticanonical divisors. The adjunction formula immediately shows that $K_{M_{i}}=0, i=1,2$, and so $M_{1}$ and $M_{2}$ have zero Kodaira dimension. While for $M_{1}$ it is easy to see that it can be chosen to be smooth, simply connected and with no torsion in cohomology, Gross shows [11, 21] that the same holds for $M_{2}$. Moreover, the two 3-folds have the same topological invariants (the second cohomology group, the Euler characteristic, the cubic form, and the first Pontrjaghin class), and so (cf. [19]), are diffeomorphic. To show that $M_{1}$ and $M_{2}$ are not deformation equivalent, note that $M_{2}$ contains a smooth rational curve with normal bundle $\mathcal{O}(-1) \oplus \mathcal{O}(-1)$, which is stable under the deformation of the complex structure, while $M_{1}$ does not. Obviously, $M_{1}$ and $M_{2}$ have the same Chern numbers. By blowing them up simultaneously at $k$ distinct points, we obtain infinitely many pairs of diffeomorphic, projective threefolds of zero Kodaira dimension, and with the same Chern numbers. 


\section{CONCluding REMARKS}

1. Let $M$ and $M^{\prime}$ be any of the pairs of complex surfaces discussed in the previous two sections. A simple inspection shows that they are not spin, and so, their intersection forms will have the form $m\langle 1\rangle \oplus n\langle-1\rangle$. By a result of Wall [23], if $m, n \geq 2$, the intersection form is transitive on the primitive characteristic elements of a fixed square. Since, $c_{1}$ is characteristic, if it is primitive too, we can assume that the homotopy equivalence $f: M \rightarrow M^{\prime}$ given by an automorphism of such an intersection form will carry the first Chern class of $M^{\prime}$ into the first Chern class of $M$. But this implies that the $h$-cobordism constructed between $X=M \times \Sigma$ and $X^{\prime}=M^{\prime} \times \Sigma$ also preserves the first Chern classes.

Following Ruan [20], we can arrange our examples such that $c_{1}$ is a primitive class. In the cases when $b_{+}>1$, which is equivalent to $p_{g}>0$, it follows that there exists a diffeomorphism $F: X \rightarrow X^{\prime}$ such that $F^{*} c_{1}\left(X^{\prime}\right)=c_{1}(X)$, where $F^{*}: H^{2}\left(X^{\prime}, \mathbb{Z}\right) \rightarrow H^{2}(X, \mathbb{Z})$ is the isomorphism induced by $F$. In these cases our theorems provide either examples of pairs of diffeomorphic Kähler threefolds, with the same Chern classes, but with different Kodaira dimensions, or examples of pairs of non-deformation equivalent, diffeomorphic Kähler threefolds, with the same Chern classes and of the same Kodaira dimension.

However, in some cases we are forced to consider surfaces with $b_{+}=1$. In these cases it is not clear whether one can arrange the $h$-cobordisms constructed between $X=M \times \Sigma$, and $X^{\prime}=M^{\prime} \times \Sigma$ also preserves the first Chern classes.

2. With our method it is impossible to provide examples of diffeomorphic 3 -folds of Kodaira dimensions $(0,3)$ and $(-\infty, 0)$. In the first case, our method fails for obvious reasons. In the second case, the reason is that for a projective surface of Kodaira dimension $-\infty$, the geometric genus $p_{g}$ is 0 , while for a simply connected projective surface of Kodaira dimension $0, p_{g} \neq 0$. Thus, any two surfaces of these dimensions will have different $b_{+}$, which is preserved under blow-ups. So, no pair of projective surfaces of these Kodaira dimensions can be $h$-cobordant. However, this raises the following question.

Question 5.1. Are there examples of pairs of diffeomorphic, projective 3-folds $\left(M, M^{\prime}\right)$ of Kodaira dimensions $(0,3)$ or $(-\infty, 0)$ ?

Most of the examples exhibited here have the fundamental group of a Riemann surface. Natural questions to ask would be the following.

Question 5.2. Are there examples of diffeomorphic, simply connected, complex, projective 3 -folds of different Kodaira dimension?

Question 5.3. Are there examples of projective, simply connected, diffeomorphic, but not deformation equivalent 3-manifolds with the same Kodaira dimension?

As we showed, the answer is yes when the Kodaira dimensions are $-\infty$ or 0 , but we are not aware of such examples in the other cases.

\section{ACKNOWLEDGEMENTS}

The author would like to thank his thesis advisor Claude LeBrun for suggesting these problems and Lowell Jones for some illuminating discussions about the Whitehead groups. 


\section{REFERENCES}

[1] R. BARLOW, A simply connected surface of general type with $p_{g}=0$, Invent. Math., 79 (1985), 293-301. MR0778128 (87a:14033)

[2] W. Barth, C. Peters, A. van de Ven, Compact Complex surfaces, Ergebnisse der Mathematik und ihrer Grenzgebiete (3), 4, Springer Verlag, Berlin, 1984. MR0749574 (86c:32026)

[3] F. Catanese, Surfaces with $K^{2}=p_{g}=1$ and their period mapping, in Algebraic Geometry (Proc. of Summer Meeting, Univ. of Copenhagen, Copenhagen, 1978), Lecture Notes in Math., 732, Springer, Berlin, 1979, 1-29. MR0555688 (81c:14018)

[4] F. Catanese, C. LeBrun, On the scalar curvature of Einstein manifolds, Math. Res. Lett., 4 (1997), 843-854. MR 1492124 (98k:53057)

[5] S.K. Donaldson, Irrationality and the h-cobordism conjecture, J. of Diff. Geom., 26 (1987), 141-168. MR0892034 (88j:57035)

[6] F.T. Farrell, L.E. Jones, Rigidity in geometry and topology, Proceedings of the International Congress of Mathematicians, Vol. I, II (Kyoto, 1990), Math. Soc. Japan, Tokyo 1991, 653-663. MR1159252 (93g:57041)

[7] M. Freedman, The topology of four manifolds, J. of Diff. Geom., 17 (1982), 357-454. MR1201584 (94b:57021)

[8] R. Friedman, J.W. Morgan Algebraic surfaces and Seiberg-Witten invariants, J. Alg. Geom., 6 (1997), 445-479. MR1487223 (99b:32045)

[9] R. Friedman, J.W. Morgan Smooth four-manifolds and complex surfaces, Ergebnisse der Mathematik und ihrer Grenzgebiete (3), 27, Springer Verlag, Berlin, 1994. MR1288304 (95m:57046)

[10] R. Friedman, Z. Qin, The smooth invariance of the Kodaira dimension of a complex surface, Math. Res. Lett., 1 (1994), 369-376. MR.1302651 (95k:57039)

[11] M. Gross, The deformation space of Calabi-Yau n-folds with canonical singularities can be obstructed, in Mirror Symmetry Vol. II, AMS/IP. Stud. Adv. Math. 1, Amer. Math. Soc., Providence, RI, 1997, 401-411. MR1416344 (98a:14054)

[12] K. Kodaira, On homotopy K3 surfaces, Essay on Topology and Related Topics (dedicated to G. de Rham), Springer, New York, 1970, 58-69. MR0262545(41:7151)

[13] K. KodaIra, On stability of compact submanifolds of complex manifolds, Amer. J. Math., 85 1963, 79-94. MR0153033 (27:3002)

[14] J. Kollár, S. MorI, Classification of three-dimensional flips, J. Amer. Math. Soc., 5 (1992), 533-703. MR.1149195 (93i:14015)

[15] C. LeBrun, Topology versus Chern numbers for complex 3-folds, Pacific J. Math., 191 (1999), 123-131. MR1725466 (2000h:32023)

[16] M. Manetti, On the moduli space of diffeomorphic surfaces, Invent. Math., 143 (2001), 2976. MR $1802792(2001 \mathrm{j}: 14050)$

[17] J. Milnor, Lectures on the h-cobordism theorem, Princeton University Press, Princeton, NJ, 1965. MR0190942 (32:8352)

[18] J. Milnor, Whitehead torsion, Bull. Amer. Math. Soc., 72 (1966), pp. 356-426. MR0196736 $(33: 4922)$

[19] Ch. OKonek, A. VAn de Ven, Cubic forms and complex 3-folds, Enseign. Math., 41 (1995), 297-333. MR:1365849 (97b:32035)

[20] Y. RuAn, Symplectic topology on algebraic 3-folds, J. of Diff. Geom., 39 (1994), 215-227. MR.1258920 (95a:14037)

[21] Y. Ruan, Topological sigma model and Donaldson-type invariants in Gromov theory, Duke Math. J., 83 (1996), 461-500. MR.1390655 (97d:58042)

[22] F. Waldhausen, Algebraic K-theory of generalized free products III, IV, Ann. of Math. (2), 108 (1978), 205-256. MR0498808 (58:16845b)

[23] C.T.C. WALL, On the orthogonal groups of unimodular quadratic forms, Math. Ann., 147 (1962), 328-338. MR0138565 (25:2009)

[24] C.T.C. WALL, On simply connected 4-manifolds, J. of London Math. Soc., 39 (1964), 141149. MR0163324 (29:627)

Department of Mathematics, SUny at Stony Brook, Stony Brook, New York 11794

E-mail address: rares@math.sunysb.edu 